Relations industrielles

Industrial Relations

\title{
Petit dictionnaire de droit québécois et canadien, par Me Dominique Pagé, Montréal, Fides, 1975, 167 p.
}

\section{Gérard Dion}

Volume 30, numéro 4, 1975

URI : https://id.erudit.org/iderudit/028673ar

DOI : https://doi.org/10.7202/028673ar

Aller au sommaire du numéro

Éditeur(s)

Département des relations industrielles de l'Université Laval

ISSN

0034-379X (imprimé)

1703-8138 (numérique)

Découvrir la revue

Citer ce compte rendu

Dion, G. (1975). Compte rendu de [Petit dictionnaire de droit québécois et canadien, par Me Dominique Pagé, Montréal, Fides, 1975, 167 p.] Relations industrielles / Industrial Relations, 30(4), 804-805.

https://doi.org/10.7202/028673ar

Tous droits réservés (C) Département des relations industrielles de l'Université Laval, 1975
Ce document est protégé par la loi sur le droit d'auteur. L'utilisation des services d'Érudit (y compris la reproduction) est assujettie à sa politique d'utilisation que vous pouvez consulter en ligne.

https://apropos.erudit.org/fr/usagers/politique-dutilisation/ 
of the Council. Further, the author makes continual reference to statements of various members of the Council without identifying either the nature of their duties on the Council or their position in either the government or employee organization hierarchy. Accordingly, the volume would have less utility for those who are not familiar with the industrial relations system in the Public Service. However, in view of the dearth of literature on the federal industrial relations environment, the author has made a significant contribution. His work should appeal to specialists and, to a lesser degree, a general audience interested in industrial relations in the Federal Public Service.

\section{A. Gray GILLESPIE}

Staff Relations Officer

Canadian Penitentiary Service

Personnel Management, A Situational

Approach, par David P. Crane, Belmont, Wadsworth Publishing, 1974, $572 \mathrm{p}$.

Voici un livre complet sur la direction du personnel, avec une optique différente. La philosophie de l'auteur reflète l'environnement dynamique des entreprises et des travailleurs, dans lequel on peut difficilement appliquer des principes immuables. En conséquence, le directeur du personnel ou le gérant doit analyser la situation pour y déceler les variables humaines, techniques et organisationnelles susceptibles de solution ner les problèmes de ses employés.

Le premier chapitre de l'ouvrage souligne le rôle significatif de l'administration du personnel dans les organisations modernes, en tenant compte des changements socio-économiques récents. Par la suite, quelques facteurs de la productivité du travailleur sont identifiées; cependant, l'auteur tend à simplifier ce sujet, puisque les travaux de Herzberg et de Sutermeister démontrent la complexité des facteurs dans l'efficacité des employés.

Le troisième chapitre met en évidence l'importance grandissante de politiques et de plans bien articulés pour la gestion des entreprises; plusieurs exemples illustrent adéquatement la nature et les conséquences de ces politiques.

Par la suite, on retrouve les fonctions classiques, telles que la sélection, le placement, le développement et la rémunération du personnel. Trois chapitres sont consacrés aux relations patronales-syrdicales, en mettant l'accent sur l'interaction des facteurs influençant les négociations collectives et les différends de travail. Enfin, le chapitre 20 identifie les changements probables dans l'avenir et souligne les secteurs stratégiques pour améliorer la «qualité de la vie de travail».

Bien que ce livre reprenne les mêmes thèmes que les ouvrages populaires en direction du personnel (v.g. Pigors \& Myers, McFarland, Megginson), il insiste sur l'analyse adéquate des situations concrètes pour diagnostiquer un problème avant de prendre une décision administrative; à cet effet, l'utilisation de problèmes et d'études de cas dans les chapitres vise à développer cette habileté chez le lecteur. Il convient de noter le langage simple de l'auteur, l'utilité d'une soixantaine de tableaux, ainsi que la présentation soignée du livre. Bref, c'est un ouvrage complet pour l'étudiant et pour le praticien qui veut mettre à jour ses connaissances.

\section{Jean LADOUCEUR}

\section{Université de Moncton}

\section{Petit dictionnaire de droit québécois et} canadien, par Me Dominique Pagé, Montréal, Fides, 1975, 167 p.

Cet ouvrage présente les définitions des termes légaux les plus courants dans les domaines juridiques suivants répartis en treize chapitres d'inégale importance: généralités, administration de la justice, droit civil, procédure civile, droit commercial, loi des compagnies d'assurance, droit municipal, droit ouvrier (sic), droit pénal ou criminel, droit constitutionnel et international, loi des faillites et droit aérien.

On comprendra que couvrir un champ aussi vaste en moins de cent cinquante pages est un défi peu ordinaire. On ne sera donc pas surpris de rester sur son appétit devant certaines définitions et de regretter certaines omissions, comme par exemple, injonction. En matière du travail, il n'y a que treize termes. L'auteur parle de "droit ouvrier», de "conflit ouvrier» qui sont des expressions dés:è̀tes. La négociation est bien davantage qu'un «moyen de régler un conflit ouvrier entre employeur et employés 
par discussions entre les parties intéressées ».

On pourrait encore chicaner l'auteur dans sa première définition du mot droit lorsqu'il affirme "Morale appliquée, transposée sur le plan social». Il y a ainsi beaucoup de simplifications.

Il faut dire que l'ouvrage ne s'adresse pas à des spécialistes, mais plutôt au grand public qui y trouvera une informatian concise et gếnérale sur des termes dont il a de plus en plus besoin de connaître les éléments essentiels.

\section{Gérard DION}

\section{Université Laval}

Industrialism and Industrial Man Reconsidered, by John T. Dunlop, Frederick H. Harbison, Clark Kerr, Charles A. Myers, Final Report for the Inter-University Study of Human Resources in National Development, Princeton, N.J., 1975, 99 p.

Ce petit volume contient les résultats d'une gigantesque étude entreprise en 1952, d'abord informellement, puis d'une façon systématique, par quatre éminents spécialistes en relations industrielles, Messieurs John T. Dunlop, Frederick $\mathrm{H}$. Harbison, Clark Kerr et Charles Myers. Ces quatre professeurs rattachés à différentes universités américaines obtinrent diverses subventions et fondèrent l'Inter-University Study of Labor Problems in Economic Development qui, en plus d'inclure toute une armée de chercheurs nord-américains, s'assura la collaboration de plusieurs autres spécialistes de tous les continents. $\mathrm{Au}$ total, plus de 36 volumes et 42 tirés-à-part ont été générés par cette équipe de chercheurs qui, au cours de la période 1953-1973, impliqua pas moins de 90 personnes.

Les hypothèses initiales furent énoncées dans un article paru en 1955 dans la Revue internationale du travail et qui s'intitulait: "The Labour Problem in Economic Development: A Framework for Reappraisal ». Le thème central développé par les auteurs était que les analyses classiques formulées par Marx, les Webbs, Hoxie, Perlman et bien d'autres présentaient une interprétation incomplète des phénomènes de relations de travail liés au développement économique en les associant uni- quement à l'évolution du capitalisme cccidental. Selon les quatre experts, un cadre de référence plus large que le régime de production capitaliste devait être utilisé pour expliquer la nature du réseau de règles («web or rules») qui se développe dans les pays industrialisés ou en voie de l'être.

Ce cadre de référence faisait appel à l'inter-action de trois agents, les employeurs, les travailleurs et leurs organisations ainsi que l'Etat et il donna lieu à la publication du célèbre Industrial Relations Systems par Dunlop en 1958.

L'intérêt dans l'approche utilisée par les quatre auteurs réside dans le fait qu'ils ont conduit (ou fait conduire) toute une série d'études dans plusieurs pays présentant des degrés d'industrialisation et des régimes politiques fort différents.

Les résultats de ces études furent d'abord publiés en 1960 dans un volume intitulé Industrialism and Industrial Man revisé en 1964 et réédité en 1973. Le présent volume consiste en une reconsidération des conclusions formulées antérieurement mais, dans l'ensemble, les auteurs continuent d'adhérer aux principes fondamentaux élaborés au cours de leurs premières études.

Les principes dans lesquels Dunlop, Harbison, Kerr et Myers continuent de croire fermement sont les suivants: 1. L'industrialisation possède une logique inhérente indépendamment des pays dans lesquels elle s'opère; 2 . La révolte ouvrière n'est pas un aspect dominant de la plupart des phénomènes d'industrialisation; 3. Une caractéristique importante du processus d'industrialisation est la montée d'une «élite 》 qui en détermine l'orientation; $A$. Un consensus minimum, accepté ou imposé, concernant les conditions générales du système de relations industrielles est indispensable au progrès économique; 5 . Le rôle des dirigeants («managers'») est primordial et, avec l'industrialisation, ceux-ci deviennent moins paternalistes et plus technocratiques; 6 . Les travailleurs organisés sont une force plus conservatrice que révolutionnaire; 7. L'Etat a tendance à intervenir de plus en plus dans le système de relations industrielles; 8 . Les ressources humaines constituent les forces les plus dynamiques dans le processus d'industrialisation et toute 